\title{
Prognostic Value of Procalcitonin in Pneumonia among Patients Admitted to Intensive Care Unit.
}

\author{
Deok Hee Kim ${ }^{1}$, Hae Won Jung ${ }^{2}$, Hyung Koo Kang ${ }^{3}$ \\ ${ }^{1}$ Department of Pulmonology, Asan Choongmu Hospital, Asan, Korea \\ ${ }^{2}$ Department of Cardiology, Daegu Catholic University Medical Center, Daegu, Korea \\ ${ }^{3}$ Department of Internal Medicine, Inje University Ilsan Paik Hospital, Goyang, Korea
}

Objectives: Pneumonia is one of the leading causes of death in the intensive care unit (ICU). Many biomarkers for predicted prognosis have been suggested; among these, procalcitonin (PCT) is known to increase in cases of bacterial infection. However, there have been many debates regarding whether PCT is an appropriate prognostic marker for pneumonia. Therefore, we investigated whether PCT can serve as a biomarker for pneumonia, and compared it with CURB-65, which is a known tool for predicting the prognosis of pneumonia. Methods: Levels of PCT and CURB-65 scores were compared between 30-day non-survival $(\mathrm{n}=30)$ and survival ( $n=101)$ patients. Relationships between PCT and CURB-65 were determined by using linear regression analysis, as well as by using receiver operating characteristic (ROC) curve analysis and calculation of the area under the curve (AUC). High and low PCT groups were compared.

Results: High PCT and high CURB-65 score were positively associated with 30-day mortality. For the prediction of 30-day mortality, initial PCT and CURB-65 exhibited AUCs of 0.63 and 0.66; these were not significantly different $(P=0.132)$. We found that the high PCT group had a higher rate of initial treatment failure $(91 \%$, $P=0.004)$.

Conclusions: Initial PCT can be a prognostic biomarker for mortality in severe pneumonia, similar to the CURB-65 score. Initial high PCT was positively associated with initial treatment failure.

Key Words: CURB-65, Mortality, Pneumonia, Procalcitonin, Prognosis

Pneumonia is one of the leading causes of mortality in intensive care units. ${ }^{1}$ Community-acquired pneumonia (CAP) is associated with a mortality rate of approximately $20 \%$ for patients admitted to hospitals in the United Kingdom; nosocomial pneumonia has a higher mortality rate than CAP. ${ }^{2,3}$ Fast recognition of severe infections and prompt ini- tiation of therapeutic regimens might decrease mortality. Empiric pathogen recognition and microbiologic pathogen recognition are likely to be helpful in choosing the appropriate antibiotics. ${ }^{4} \mathrm{~A}$ growing number of clinical research studies have identified blood biomarkers that may reveal additional information regarding the prognosis of pa-
Corresponding Author: Hyung Koo Kang, Department of Internal Medicine, Inje University Ilsan Paik Hospital, 170, JooHwa-ro, Ilsan-Seo-gu, Goyang 10380, Korea

Tel: +82-31-740-0114 Fax: +82-31-910-7108 E-mail: inspirit26@paik.ac.kr

\section{(1) (2)}

Articles published in Kosin Medical Journal are open-access, distributed under the terms of the Creative Commons Attribution Non-Commercial License (http://creativecommons.org/licenses/by-nc/4.0/) which permits unrestricted non-commercial use, distribution, and reproduction in any medium, provided the original work is properly cited.
Received: Sep. 07, 2018

Revised: Nov. 04, 2018

Accepted: Nov. 21, 2018 
tients with pneumonia. ${ }^{5-8}$

Procalcitonin (PCT) is a useful marker for the diagnosis of bacterial infection"; this 116-amino-acid precursor is the prohormone of calcitonin, which is secreted by $\mathrm{C}$-cells of the thyroid gland in response to hypercalcemia. Notably, it mirrors the severity of infection and has emerged as the most studied and promising blood marker for risk stratification of patients with pneumonia. Concentrations of PCT tend to be higher in patients with pneumonia who exhibit more severe infections. $^{10,11}$ Several risk scores, such as the pneumonia severity index (PSI) and the confusion, urea nitrogen, respiratory rate, blood pressure, age $\geq 65$ years (CURB-65) score, can be used to assess the severity of pneumonia and predict mortality. In particular, CURB-65 is a well-known set of criteria that is recommended by the British Thoracic Society for the assessment of severity of pneumonia. It can also be a useful tool for estimating the prognosis of hospital-acquired pneumonia (HAP). ${ }^{12}$ However, there have been many debates regarding whether PCT is an appropriate prognostic marker for pneumonia. Therefore, we investigated whether PCT can serve as a biomarker for patients with pneumonia requiring intensive care unit treatment, and compared it with CURB-65.

\section{MATERIALS AND METHODS}

\section{Study Patients}

We retrospectively conducted an observational cohort study at a single center (Ilsan Paik hospital in Goyang-si, South Korea) between March 2015 and May 2017. Patients were screened by the following Korean Standard Classification of Disease-7 codes: J18.0-J18.9. Medical records and radiological findings were reviewed to confirm the diagnosis of pneumonia by the following criteria: the development of a new opacity and 2 of the following 4 criteria met. ${ }^{13}$

1) Fever or hypothermia (Body temperature $\geq$ $38^{\circ} \mathrm{C}$ or $\left.\leq 35.0^{\circ} \mathrm{C}\right)$

2) Leukocytosis (WBC $\geq 10000 / \mu \mathrm{L}$ ) or leukopenia $(\leq 4000 / \mu \mathrm{L})$

3) Newly developed respiratory symptom (cough, sputum, pleuritic chest pain, and/or dyspnea)

4) Auscultation abnormality (crackles)

Primary or metastatic lung cancer, pulmonary edema, pulmonary embolism, and pulmonary tuberculosis were excluded. We also excluded the following patients:

1) Those who were transferred from another hospital after or during treatment (to be nearer to their hometowns or to undergo renal replacement therapy)

2) Immunocompromised patients (e.g., those with neutropenia after chemotherapy or human immunodeficiency virus infection)

3) Pneumonia in HAP, including patients who were hospitalized $\geq 2$ days within 90 days of infection.

A total of 131 patients were included in the analysis. Among these patients, 30 died within 30 
days. We divided patients into two groups on the basis of 30 day-survival (Fig. 1). We compared clinical characteristics, comorbidities, 30-day mortality, microorganisms identified from sputum or blood, severity by initial CURB-65, and the use of initial mechanical ventilator or initial vasopressor.

Measurement

Patient medical records were reviewed to obtain data regarding patient demographic characteristics, underlying diseases, initial CURB-65, comorbidities (including diabetes mellitus, chronic obstructive pulmonary disease, malignant neoplasm, and chronic renal disease), causative microorganisms for pneumonia, initial vasopressor use, initial mechanical ventilator use, initial C-reactive protein (CRP) levels, initial PCT, result of treatment (treatment failure or not), and estimated glomerular filtration rate (eGFR). Serum PCT concentrations were measured by using an electrical chemiluminescence assay (cobas e 411,
Roche Diagnostics, Indianapolis, IN, USA), with an analytical measurement range of 0.00-0.00. Elevated serum PCT levels were defined when serum PCT levels were > $0.25 \mathrm{ng} / \mathrm{dL}$. White blood cell (WBC) counts and CRP levels were measured with laser flow cytometry and immunoturbidimetric assays.

\section{Definition}

Definition of abnormal white blood cell count (WBC): we defined the abnormal WBC count as any count not in the normal range; normal WBC range $(4-10 \times 109$ cells/L) was applied to all age groups. ${ }^{14}$

Definition of initial antibiotics failure: failure of initial antibiotics therapy was defined as death during initial treatment or change of antibiotics from the initial agents to other agents after 48 hours due to clinical instability. ${ }^{15}$

Definition of low eGFR, based on the Modification of Diet in Renal Disease (MDRD) equation, was lower

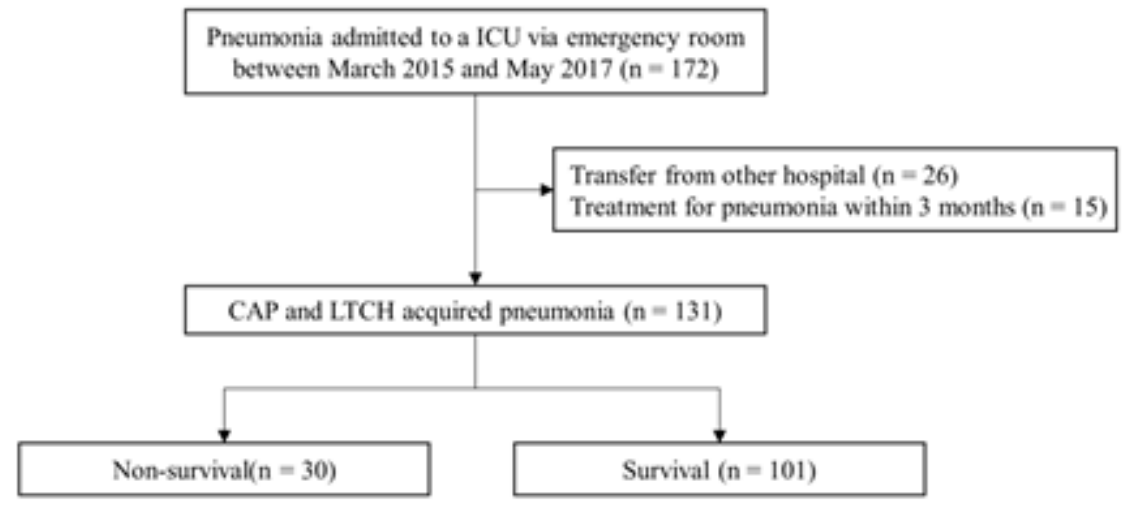

Fig. 1. Selection process for patients of pneumonia ICU, intensive care unit; CAP, community acquired pneumonia; LTCH, long term care hospital 
than $90 \mathrm{~mL} / \mathrm{min} / 1.73 \mathrm{~m}^{2}{ }^{16}$

Microbiology

Sputum, tracheal aspirate, bronchial washing secretions, and blood samples were investigated for microbial analysis. Pathogens were considered to be identified when a predominant microorganism was detected from sputum culture and/or sputum PCR. Blood cultures were considered as pathogens if there was no other infection source available for a positive culture; we excluded positive cultures in which contamination was suspected.

Statistical analysis

Descriptive analysis was performed to evaluate baseline characteristics of the patients. Comparison of continuous variables was performed by the Mann-Whitney U-test between two groups. Categorical variables were compared using the Pearson $\chi^{2}$ test. Baseline characteristics are presented as medians and interquartile ranges (IQRs) for continuous variables and as numbers (\%) for categorical variables. To identify independent predictive factors associated with 30-day mortality, we performed multivariable logistic regression analysis by using these variables: PCT, high CURB-65, sex, and age, as measured by the estimated odds ratio (OR) with 95\% confidence interval (CI). Receiver operating characteristic (ROC) curve analysis and calculation of the area under the curve (AUC) were performed. Correlations between PCT and CURB-65 were examined by using the Spearman test. Analyses were performed by using the Statistical Package for the
Social Sciences (SPSS) version 21.0 (SPSS; Chicago, IL, USA) and SAS (version 9.4, Cary, NC, USA).

\section{RESULTS}

\section{Baseline characteristics}

The baseline characteristics of study patients are summarized in Table 1. Compared to the survival group, there were more males in the non-survival group $(76.7 \%$ [23/30] vs. $55.4 \%$ [56/101], $P=0.028$ ); there were no differences in age or comorbidities. Notably, the non-survival group showed more frequent initial vasopressor use (80.0\% [26/30] vs. $60.4 \%$ [61/101], $P=0.005)$ and initial mechanical ventilator use $(73.3 \%$ [22/30] vs. $53.5 \%$ [54/101], $P=0.041)$. The mean age of the non-survival group was 76.3 (65.5-86.0) years; $76.7 \%$ of the non-survival patients were male $(P=0.028)$. The two groups were similar in baseline characteristics, except patients in the non-survival group had higher CURB-65 scores $(P=0.024)$ and a history of malignant neoplasm $(P=0.040)$. Average PCT levels were not significantly different (35.2 vs. 9.12, $P=0.129$ ), but the non-survival group exhibited a higher PCT level ( > $0.25 \mathrm{ng} / \mathrm{dL}$ ) than the survival group (96.2\% vs. $77.9 \%, P=0.025)$. The 30 -day mortality in the present study was $22.9 \%$ (Table 1 ).

CURB-65 and PCT as predictive factors of 30-day mortality

We performed univariate and multivariate anal- 
Table 1. Baseline characteristics of 131 patients admitted to an intensive care unit with pneumonia.

\begin{tabular}{llll}
\hline Characteristics & $\begin{array}{c}\text { Death } \\
\text { (within } 30 \text { days) } \\
(\mathrm{n}=30) 22.9 \%\end{array}$ & $\begin{array}{c}\text { Survived } \\
(\mathrm{n}=101) 77.1 \%\end{array}$ & $P_{\text {value }}$ \\
\hline Age, year & $76.3(65.5-86.0)$ & $74(67-83)$ & 0.402 \\
Male & $23(76.7 \%)$ & $56(55.4 \%)$ & 0.028 \\
Comorbidities & $11(36.7)$ & $33(32.7)$ & 0.421 \\
Diabetes mellitus & $8(26.7)$ & $24(23.8 \%)$ & 0.458 \\
COPD & $6(20.0 \%)$ & $7(6.9 \%)$ & 0.046 \\
Malignant neoplasms & $3(10.0 \%)$ & $5(5.0 \%)$ & 0.266 \\
Chronic renal disease & & & \\
Clinical parameters & $24(80.0 \%)$ & $59(58.4 \%)$ & 0.024 \\
CURB-65 score $\geq 3$ & $24(80.0 \%)$ & $79(78.2 \%)$ & 0.528 \\
Microorganism identified & $26(86.7 \%)$ & $61(60.4 \%)$ & 0.005 \\
Initial vasopressor & $22(73.3 \%)$ & $54(53.5 \%)$ & 0.041 \\
Initial mechanical ventilation & & & \\
Laboratory findings & & $13.27(7.29-18.16)$ & 0.020 \\
White blood cell, 1000/mm3 & $9.66(3.95-14.52)$ & $73(72.3 \%)$ & 0.352 \\
Abnormal WBC count & $20(66.7 \%)$ & $9.12(0.33-6.25)$ & 0.129 \\
Procalcitonin, ng/dL & $35.2(0.87-37.8)$ & $13.7(4.8-21.7)$ & 0.385 \\
C-reactive protein, mg/dL & $15.6(4.1-25.0)$ & $1.35(0.68-1.56)$ & 0.767 \\
Creatinine, mg/dl & $1.42(0.72-2.05)$ & $0.89(0.50-0.92)$ & 0.687 \\
Total bilirubin, mg/dl & $0.97(0.60-1.27)$ & $67(66.3 \%)$ & 0.025 \\
High procalcitonin(>0.25ng/dl) & $25(96.2 \%)$ & & \\
\hline
\end{tabular}

Data are presented as number (\%) or median (interquartile range).

COPD, chronic obstructive lung disease

Table 2. Univariate and multivariate study for Odd ratio of 30 day mortality.

\begin{tabular}{lcccc}
\hline \multirow{2}{*}{ Characteristics } & \multicolumn{2}{c}{ Univariate } & \multicolumn{2}{c}{ Multivariate } \\
\cline { 2 - 5 } & OR $(95 \% \mathrm{Cl})$ & $P_{\text {value }}$ & $\mathrm{OR}(95 \% \mathrm{Cl})$ & $P_{\text {value }}$ \\
\hline Procalcitonin & $1.021(1.002-1.041)$ & 0.035 & $1.020(1.000-1.041)$ & 0.047 \\
CURB-65 score $\geq 3$ & $2.847(1.071-7.573)$ & 0.036 & $1.573(0.513-4.821)$ & 0.428 \\
Male & $2.640(1.039-6.709)$ & 0.041 & $3.073(1.036-9.115)$ & 0.043 \\
Old age & $1.503(0.650-3.480)$ & 0.341 & $1.864(0.667-5.208)$ & 0.235 \\
\hline
\end{tabular}

ysis by using risk factors for mortality (Table 2). In the multivariate analysis, PCT (OR 1.020 (1.000-1.041), $P=0.04$ ), and male sex (OR 3.073 (1.036-9.115), $P=0.043$ ) were found to be independent predictors of mortality. When the non-survival group was compared with the survival group, high CURB-65 score ( $\geq 3)(80.0 \%$ [24/30] vs. $58.4 \%[59 / 101], P=0.024)$ and high PCT ( $>$
$0.25 \mathrm{ng} / \mathrm{dL})(96.2 \%[25 / 30]$ vs. $66.3 \%$ [67/101], $P$ $=0.025)$ were more frequent. In ROC curve analysis (Fig. 2) for prediction of 30-day mortality, initial PCT and CURB-65 at admission time had AUCs of 0.631 (95\% CI, 0.504-0.758; $P=0.043$ ) and 0.662 (95\% CI, 0.546-0.778; $P=0.014$ ). There was no significant difference between PCT and CURB-65 $(P=0.132)$ (Table 3$)$. Our study showed that high 


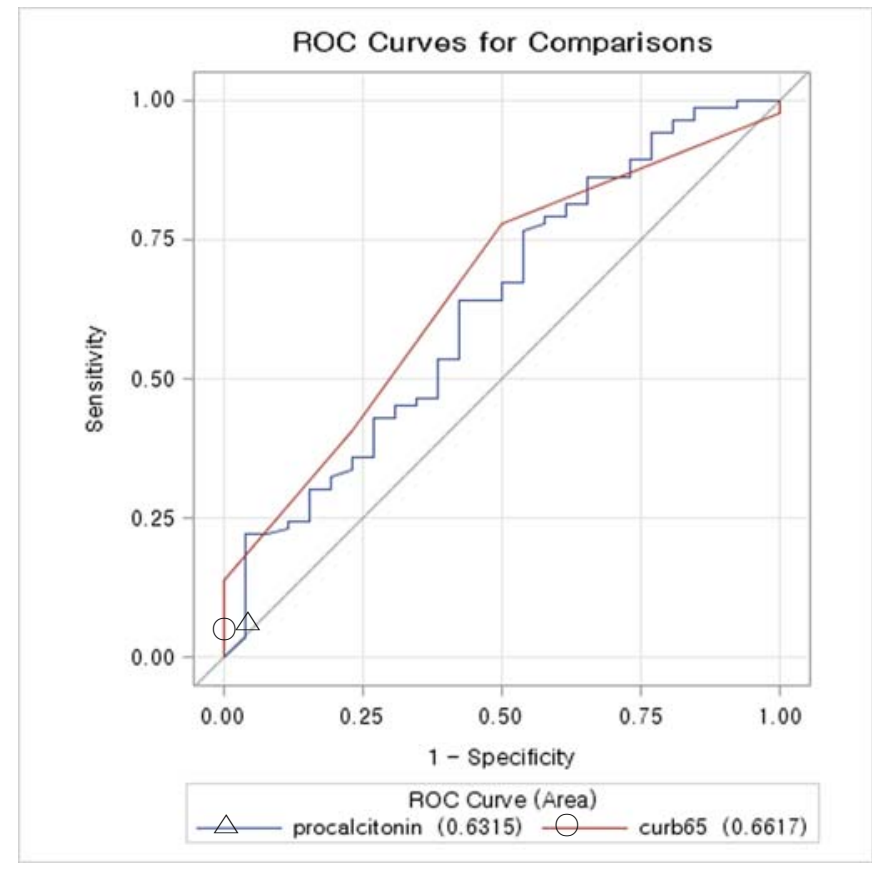

Fig. 2. Area under the curve (AUC) of procalcitonin and CURB-65 is greater than 0.5 , with no significant difference.

$\mathrm{ROC}$, receiver operating characteristic

Table 3. Results of receiver operating characteristic (ROC) curve analysis

\begin{tabular}{lcc}
\hline \multirow{2}{*}{ Characteristics } & \multicolumn{2}{c}{ Mortality within 30 days } \\
\cline { 2 - 3 } & AUC $(95 \% \mathrm{Cl})$ & $P$ value \\
\hline Procalcitonin & $0.631(0.504-0.758)$ & 0.043 \\
CURB-65 & $0.662(0.546-0.778)$ & 0.014 \\
Procalcitonin versus CURB-65 & & 0.132 \\
\hline
\end{tabular}

PCT is related to initial treatment failure (Table 4). Subgroup analysis of the high PCT group showed an association with high CURB-65 ( $\geq$ 3) (Table 5). Initial mechanical ventilator use $(P$ $=0.009$, vasopressor use $(P=0.011)$ and leukopenia $(P=0.026)$ were related to initial treatment failure. However, low MDRD eGFR $(P=0.183)$, whether causative organism was identified $(P=$ 0.094), and old age ( > 74) were not related to initial treatment failure.

\section{DISCUSSION}

A previous study has shown that PCT can be prognostic biomarker for CAP (AUC 0.60, CI: 0.52-0.67), but has a lower prognostic value than CURB-65 $(P=0.001) .{ }^{17}$ One meta-analysis of the prognostic value of PCT in pneumonia ${ }^{18}$ showed that elevated PCT level was associated with an increased risk of mortality (RR 4.18, 95\% CI: 3.19-5.48). PCT is thus an emerging biomarker for the diagnosis of bacterial infection. 
Table 4. Cross-sectional analysis between initial treatment failure and high procalcitonin

\begin{tabular}{lclc}
\hline Characteristics & $\begin{array}{c}\text { Initial treatment failure } \\
(61,54.4 \%)\end{array}$ & $\begin{array}{c}\text { Non-failure } \\
(51,39.1 \%)\end{array}$ & $P$ value \\
\hline High procalcitonin $(>0.25 \mathrm{ng} / \mathrm{dl})$ & $56(91.8 \%)$ & $36(70.5 \%)$ & 0.004 \\
\hline
\end{tabular}

Data are presented as number (\%)

Table 5. Subgroup analysis of the high procalcitonin group.

\begin{tabular}{lccc}
\hline Characteristics & $\begin{array}{c}\text { Initial treatment } \\
\text { failure group } \\
(56,60.9 \%)\end{array}$ & $\begin{array}{c}\text { Non-failure } \\
(36,39.1 \%)\end{array}$ & $P$ value \\
\hline Old age (\rangle 74$)$ & $30(65.2 \%)$ & $16(34.8 \%)$ & 0.261 \\
Male & $41(73.2 \%)$ & $15(26.8 \%)$ & 0.021 \\
Clinical parameters & $42(75.0 \%)$ & $20(55.5 \%)$ & 0.044 \\
CURB-65 score $\geq 3$ & & & 0.011 \\
Microorganism identified & $45(80.4 \%)$ & $20(55.6 \%)$ & 0.009 \\
Initial vasopressor & $40(71.4 \%)$ & $16(44.4 \%)$ & 0.094 \\
Initial mechanical ventilation & $48(85.7 \%)$ & $26(72.2 \%)$ & 0.183 \\
Organism idendtifited & & & 0.026 \\
Laboratory findings & $34(60.7 \%)$ & $26(72.2 \%)$ & \\
Low estimated GFR< $(\langle 80)$ & $10(17.9 \%)$ & $3(8.3 \%)$ & \\
Leukopenia &
\end{tabular}

Data are presented as number (\%)

In this study, PCT had only a moderate ability to predict 30-day mortality. However, its prognostic value did not differ from that of CURB-65. This study showed that high PCT level ( >0.25 $\mathrm{ng} / \mathrm{dL}$ ) can be a risk factor for 30-day mortality. We investigated the relationship of various characteristics to initial treatment failure. Leukopenia ( $<4000 / \mu \mathrm{L})$ was related to treatment failure $(P$ $=0.026$ ), but old age ( $>75)$ and low eGFR were not. Low eGFR is known to indicate a risk of death with pneumonia, especially in young patients. ${ }^{19}$ However, our study did not find similar evidence. Initial mechanical ventilator use $(P=0.009)$, initial vasopressor use $(P=0.011)$, initial leukopenia $(P$
$=0.026$ ) were related to initial treatment failure. Thus, we suspect that these patients have more severe pneumonia, so the risk of treatment failure is higher.

PCT is a known biomarker that is elevated in bacterial infections, especially in those caused by gram-negative bacteremia. ${ }^{20}$ No research has indicated that high PCT is related to a high rate of causative organism identification. This study showed a minor tendency, but no significant difference; if the causative organism could be identified, the patient was not likely to show high PCT. The CURB-65 score is frequently used to predict the prognosis of pneumonia, both in CAP and in 
HAP. Our investigation showed that initial PCT also has prognostic value, similar to that of CURB-65.

Our study has some limitations. First, it was performed at a single center, and a small number of patients were enrolled by using a retrospective design. In addition, we did not stratify cases of pneumonia on the basis of CAP or HAP.

Initial PCT can be a prognostic biomarker for mortality in severe pneumonia. Its value is similar to that of CURB-65 score.

\section{Conflict of Interest statement}

The authors declare that they have no conflicts of interest.

\section{ACKNOWLEDGEMENT}

This research did not receive any specific grant from funding agencies in the public, commercial, or not-for-profit sectors.

\section{REFERENCES}

1. Xu J, Murphy SL, Kochanek KD, Bastian BA. Deaths: Final Data for 2013. Natl Vital Stat Rep 2016;64:1-119.

2. Leu HS, Kaiser DL, Mori M, Woolson RF, Wenzel RP. Hospital-acquired pneumonia. Attributable mortality and morbidity. Am J Epidemiol 1989;129:1258-67.

3. Masterton RG, Galloway A, French G, Street M, Armstrong J, Brown E, et al. Guidelines for the management of hospital-acquired pneumonia in the UK: report of the working party on hospital-acquired pneumonia of the British Society for Antimicrobial Chemotherapy. J Antimicrob Chemother 2008;62:5-34.

4. Woodhead M, Blasi F, Ewig S, Garau J, Huchon $G$, Ieven $M$, et al. Guidelines for the management of adult lower respiratory tract infections--full version. Clin Microbiol Infect 2011;17:E1-59.

5. Hohenthal U, Hurme S, Helenius H, Heiro M, Meurman O, Nikoskelainen J, et al. Utility of C-reactive protein in assessing the disease severity and complications of community-acquired pneumonia. Clin Microbiol Infect 2009;15:1026-32.

6. van Vugt SF, Broekhuizen BD, Lammens C, Zuithoff $\mathrm{NP}$, de Jong PA, Coenen S, et al. Use of serum $\mathrm{C}$ reactive protein and procalcitonin concentrations in addition to symptoms and signs to predict pneumonia in patients presenting to primary care with acute cough: diagnostic study. BMJ 2013;346:f2450.

7. Póvoa P, Coelho L, Almeida E, Fernandes A, Mealha R, Moreira P, et al. Early identification of intensive care unit-acquired infections with daily monitoring of C-reactive protein: a prospective observational study. Crit Care 2006;10:R63.

8. Ramírez P, Ferrer M, Marti V, Reyes S, Martinez $\mathrm{R}$, Menéndez R, et al. Inflammatory biomarkers and prediction for intensive care unit admission in severe community-acquired pneumonia. Crit Care Med 2011;39:2211-7.

9. Jain S, Sinha S, Sharma SK, Samantaray JC, Aggrawal P, Vikram NK, et al. Procalcitonin as 
a prognostic marker for sepsis: a prospective observational study. BMC Res Notes 2014;7:458.

10. Assicot M, Gendrel D, Carsin H, Raymond J, Guilbaud J, Bohuon C. High serum procalcitonin concentrations in patients with sepsis and infection. Lancet 1993;341:515-8.

11. Horie M, Ugajin M, Suzuki M, Noguchi S, Tanaka W, Yoshihara H, et al. Diagnostic and prognostic value of procalcitonin in community-acquired pneumonia. Am J Med Sci 2012;343:30-5.

12. Menéndez R, Martínez R, Reyes S, Mensa J, Filella $\mathrm{X}$, Marcos M, et al. Biomarkers improve mortality prediction by prognostic scales in community-acquired pneumonia. Thorax 2009;64:587-91.

13. Carratalà J, Fernández-Sabé N, Ortega L, Castellsagué X, Rosón B, Dorca J, et al. Outpatient care compared with hospitalization for community-acquired pneumonia: a randomized trial in low-risk patients. Ann Intern Med 2005;142:165-72.

14. Walker, HK. Clinical Methods: The History, Physical, and Laboratory Examinations. 3rd ed. Boston: Butterworths; 1990

15. Maruyama T, Fujisawa T, Okuno M, Toyoshima H, Tsutsui K, Maeda H, et al. A new strategy for healthcare-associated pneumonia: a 2-year pro- spective multicenter cohort study using risk factors for multidrug-resistant pathogens to select initial empiric therapy. Clin Infect Dis 2013;57:1373-83.

16. National Kidney Foundation. K/DOQI clinical practice guidelines for chronic kidney disease: Evaluation, classification, and stratification. Am J Kidney Dis 2002;39:\$1-266.

17. Schuetz P, Suter-Widmer I, Chaudri A, Christ-Crain M, Zimmerli W, Mueller B, et al. Prognostic value of procalcitonin in community-acquired pneumonia. Eur Respir J 2011;37:384-92.

18. Liu D, Su LX, Guan W, Xiao K, Xie LX. Prognostic value of procalcitonin in pneumonia: A systematic review and meta-analysis. Respirology 2016;21:280-8.

19. James MT, Quan H, Tonelli M, Manns BJ, Faris $\mathrm{P}$, Laupland $\mathrm{KB}$, et al. CKD and risk of hospitalization and death with pneumonia. Am J Kidney Dis 2009; $54: 24-32$.

20. Guo SY, Zhou Y, Hu QF, Yao J, Wang H. Procalcitonin is a marker of gram-negative bacteremia in patients with sepsis. Am J Med Sci 2015;349:499-504. 